\title{
Evaluation of mucosal vaccines against pneumococcal and meningococcal diseases
}

\begin{abstract}
Pneumonia and meningitis represents important infections in young children (under five years old). Vaccination has been the most important tool against these diseases. However, even with efficient vaccines, pneumococcal pneumoniae was globally equivalent to $15 \%$ of all deaths in children, and meningococcal diseases are constantly present as outbreaks in Africa. Bio-Manguinhos/Oswaldo Cruz Foundation (Brazil), supplies the 10-valent pneumococcal vaccine and meningococcal vaccines. The pneumococcal vaccine contains 10 of the more than 90 pathogen's serotypes, and meningococcal vaccine contains only one of the five serotypes presents in outbreaks. The mentioned diseases are acquired by respiratory tract and all vaccines available to these bacterial infections are administered using syringes and needles through intramuscular route. In spite of mucosal administration being an interesting and advantageous option as vaccines against respiratory acquired diseases, very few of them are present on market. It occurs because of difficulties in find adequate delivery systems and adjuvants used in formulations. Among the polymers studied as candidate for mucosal vaccines, chitosan has an emphasis as a natural cationic polysaccharide, extensively reviewed in the literature, with many applications in the pharmaceutical fields due to its favourable characteristics, such as non-toxicity, biocompatibility, biodegradability and properties as bio adhesion. Very few mucosal vaccines are available on market and are still on initial stages the studies with these bacterial infecions. In order to improve the distribution and adhesion to the vaccination for all age groups it is important to move forward and propose different options in immunization techniques.
\end{abstract}

Keywords: pneumococcal vaccine, meningococcal vaccine, mucosal vaccine chitosan
Volume 4 Issue 5 - 2017

\author{
Ana Maria Pereira Dos Santos, ${ }^{1,2}$ Isabelly \\ Santos Pereira,' Mariana Miguez,' Ana Paula \\ Corrêa Argondizzo,' Maria Helena Rocha- \\ Leão ${ }^{2}$ \\ 'Oswaldo Cruz Foundation (FIOCRUZ), Brazil \\ ${ }^{2}$ Department of Biochemistry, Federal University of Rio de \\ Janeiro, Brazil
}

Correspondence: Ana Maria Pereira Dos Santos, Oswaldo Cruz Foundation (FIOCRUZ), Bio-Manguinhos, Avenida Brasil 4365, 21045-900, Rio de Janeiro/RJ, Brazil, Tel +55 2l 3882-7180, Email anamaria@bio.fiocruz.br

Received: October 30, 2017 | Published: November 22, 2017
Abbreviations: IM, intramuscular; IN, intranasal; SC, subcutaneous; pRc/CMV-HBs, expresses the hepatitis b surface antigen; SARS-CoV, severe acute respiratoty syndrome coronavirus; HBsAg, surface antigen of hepatitis B virus; DC, dendritic cells; IgG, immunoglobulin G; IgA, immunoglobulin adapted.

\section{Introduction}

Pneumonia is an infection that affects respiratory tract and lungs and it's globally the largest infectious cause of death with 920,136 deaths in 2015 for children under five years old, representing 15\% of all obits in this age. The main infectious agent of pneumonia, the bacterium Streptococcus pneumoniae, is normally found at nose or throat and can spread by droplets from a cough or sneeze. ${ }^{1} \mathrm{~S}$. pneumoniae is capable to cause serious severe invasive disease, but it can colonize individuals that will not to present symptoms, suggesting that colonization represents the primary selective force for this bacterium. ${ }^{2}$ In the same way, meningococcal meningitis is a bacterial life-threatening disease caused mainly by Neisseria meningitidis. In Europe, the US and other developed countries, meningococcal disease incidence is typically between 1 and 10 per 100,000 population, with occasional 'hyper endemic' periods of persistent disease caused by particular strains. ${ }^{3}$ A strip of land of Sub-Saharan Africa, extending from Senegal to Ethiopia is referred as the "African Meningitis Belt", represents 430 million people at risk in 26 African countries, with recurring epidemics causing 20,000 to 200,000 cases and 2,000 to 20,000 deaths annually. ${ }^{4}$ As transmission of these diseases occurs mainly by respiratory tract, including from asymptomatic individuals carriers, mucosal immunization mechanisms must be constantly evaluated.

\section{Discussion}

\section{Mucosal vaccines}

Considering preventing diseases acquired by respiratory tract and other mucosal routes, the formation of a strong mucosal barrier can be an effective strategy, as it is the first point of contact with the host. However very few mucosal vaccines are available on the market, ${ }^{5}$ as described in (Table 1), from Lycke, published in 2012. ${ }^{6}$ Mucosal vaccination (oral or intranasal route) can favors the development of mucosal antibody and cell mediated immune response. ${ }^{6}$ Nasal experiments with recombinant protein pneumococcal vaccines presented favorable results, capable to prevent a systemic infection and generate levels of $\operatorname{IgA}$ and $\operatorname{IgG}$ in to systemic and mucosal immune mechanisms. ${ }^{7}$ Studies are also presented promisors preliminary results for meningococcal antigen, with experiments with serotype $\mathrm{C}$ conjugated formulations in chitosan. ${ }^{8}$

\section{Chitosan polymer in studies for pharmaceutical applications}

Chitosan, is a natural polysaccharide and the second most abundant after cellulose. It is composed of $\beta(1 \diamond 4)$-linked 2-acetamido-2deoxy$\beta$-D-glucose, ${ }^{9}$ formed by deacetylation of chitin. Its molecular weight and chemical modifications are responsible for physical-chemical proprieties as solubility. In acid solutions, chitosan is soluble and charged positively and neutral and alkaline, it is insoluble. ${ }^{9,10}$ Due its safety and biocompatibility for topical and oral use, chitosan was been used in several pharmaceutical applications, as constituent of matrix for encapsulation and immobilization of drugs and also as a mucoadhesive material and as a transmucosal absorption promoter. ${ }^{11}$ The positive charged particles of chitosan interact electro statically 
with sialic acid residues present on mucosal surface and it helps on antigen absorption, because affects the permeability of epithelial cells. ${ }^{12}$ These properties conferred to chitosan publications and experiments in pharmaceutical formulations (Table 2). ${ }^{13}$ Studies using a recombinant protein (PsaA, Pneumococcal surface adhesin A) as pneumococcal vaccine in intranasal formulation with chitosan showed that chitosan can be capable to enhance the mucosal immune responses of the protein (Figure 1) [14 XU 2015]. Very few studies were presented for meningococcal vaccines in mucosal administration, as the patent JP2010209122 (from 2010) that includes a formulation for an intranasal formulation using chitosan..$^{15}$ More recently is emerging a new study with a conjugated meningococcal $\mathrm{C}$ meningitis formulated in chitosan. The very preliminary results showed that the chitosan formulation was as capable to protect mice against challenge as the original formulation used in the study (Table 3 ). ${ }^{8}$

Table I Mucosal vaccines available on market

\begin{tabular}{lll}
\hline Vaccine & Trade Name (Developer) & Administration \\
\hline Influenza type A and B viruses & FluMist (Medlmmune) & Intranasal \\
\hline HINI influenza vírus (swine flu) & NASOVAC (Serum Institute of India) & Intranasal \\
\hline Rotavirus & RotaTeq (Merck); Rotarix (GlaxoSmithkline) & Oral \\
\hline Poliovirus & Many & Oral \\
\hline Salmonella typhi & Vivotif (Crucell);Ty2IA & Oral \\
\hline Vibrio cholearae & Orochol (Crucell) & Oral \\
\hline Cholera & Dukoral (Crucell); Shanchol (Shantha Biotechnics) & Oral \\
\hline
\end{tabular}

*Adapted from Lycke, 2012.

Table 2 Chitosan based mucoadesive preparation experimentally used for various drugs ${ }^{13}$

\begin{tabular}{|c|c|c|c|}
\hline Delivery System & Antigen & Administration Route & Efficacy Results \\
\hline \multirow{5}{*}{ Nanoparticles } & pRc/CMV-HBs (Plasmid) & IN & $\begin{array}{l}\text { Protective IgG levels, as well high IgA levels in nasal, salivary and vaginal } \\
\text { secretions, after } 2 \text { immunizations }\end{array}$ \\
\hline & TT & IN & $\begin{array}{l}\text { IgG levels upon } 3 \text { doses were higher than dose reported with the antigen in } \\
\text { solution, and comparable to IM alum-adsorbed vaccine }\end{array}$ \\
\hline & \multirow{3}{*}{$\begin{array}{l}\text { Antigen-encoding } \\
\text { plasmids }\end{array}$} & $\mathrm{SC} / \mathrm{IN}$ & $\begin{array}{l}\text { Plasmid encoding antigen 85B (M. tuberculosis). Co-encapsulation of another } \\
\text { plasmid encoding an autophagy-inducing factor (myc-mTOR). Strong IgG and } \\
\text { cytokine (IL-4 anf IFN- } \gamma \text { ) after SC prime and two IN boosts. }\end{array}$ \\
\hline & & IN & $\begin{array}{l}\text { Plasmid encoding pHSP65pep (M. tuberculosis). Stron antibody and T-cell } \\
\text { responses and increased protection against challenge after } 4 \text { immunizations }\end{array}$ \\
\hline & & IN & $\begin{array}{l}\text { Plasmid encoding the SARS-CoV nucleocapsid protein. Particles funcionalizad } \\
\text { with a protein vector for DC targeting (bfFP) and DC maturation stimulus } \\
\text { (aCD40) showed better targeting to DCs and increased mucosal response. }\end{array}$ \\
\hline Nanocapsules & $\mathrm{rHBsAg}$ & IM, IN & $\begin{array}{l}\text { A single IM dose of the vaccine prototype elicted similar IgG levels as two IM } \\
\text { doses of alum-adsorbed antigen. }\end{array}$ \\
\hline Liposomes & $\begin{array}{l}\text { Antigen-encoding } \\
\text { plasmids }\end{array}$ & IN & $\begin{array}{l}\text { Plasmid encoding } \mathrm{HBsAg} \text { Glycol chitosan-coated liposomes elicted } \\
\text { seroprotection and increases IgA levels in nasal, vaginal and salivary } \\
\text { secretions, compared with controls. }\end{array}$ \\
\hline
\end{tabular}

Table 3 Preliminary results using a conjugated polysaccharide from meningococcal C bacteria formulated with chitosan, after challenge with Neisseria meningitides serogroup $C$

\begin{tabular}{llll}
\hline Group & Animals on Study & Obits & Evaluation \\
\hline Original conjugated vaccine (PSC-MenC) & 10 & 0 & Tested animals remained healthy after test \\
\hline (Positive control) & & & \\
\hline Formulation Chitosan+PSC-MenC & 10 & 0 & Tested animals remained healthy after test \\
\hline Naked Chitosan (Negative control) & 5 & 3 & The survivors presented clinical symptoms of infection \\
\hline
\end{tabular}

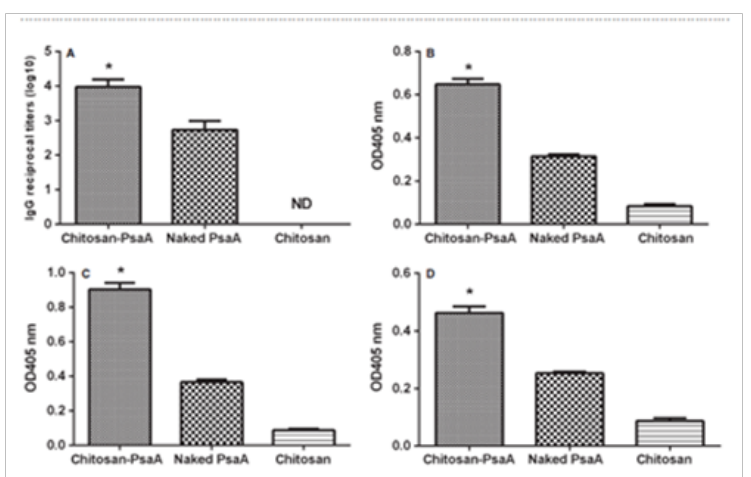

Figure I ELISA analysis of total anti-PsaA IgG (A) antibody in serum and IgA antibody in NW (B), BALF (C) and MEL (D).
The serum was detected at 1:100 dilutions, then two-fold proportion dilutions for IgG. The NW, MEL or BALF were detected for IgA without dilutions. The data from serum samples are presented geometric mean titre _ standard deviation of six mice per group. The data from NW, MEL or BALF are presented as mean OD405 _ standard deviation of six mice per group. The reciprocal titre was considered the last dilution of serum that registered an optical density of 0.10. Statistical analysis was performed using one-way ANOVA. Statistical difference between the group immunized with chitosanPsaA and that immunized with the naked PsaA $(\mathrm{P}<0.05)$ is marked with an asterisk. BALF: bronchoalveolar fluids; MEL: middle ear lavages; NW: nasal washes from. ${ }^{14}$

\section{Conclusion}

As chitosan is one of the most exploited polymers in biomedical 
science, ${ }^{10}$ and in broadly recognized by its mucohadesive properties ${ }^{12}$ ${ }^{13}$ this polymer could be considered as an opportunity to construct platforms of intranasal vaccines against pneumococcal pneumoniae and meningococcal meningitis, as they are severe infections that affects children in several countries. Results from PsaA studies ${ }^{14}$ and PSC-MenC vaccines ${ }^{8}$ on state of art, are important to reinforce mucosal vaccines for bacterial infections from respiratory tract as an alternative of immunization, exploiting the natural way of contamination of these diseases.

\section{Conflicts of interest}

The authors do not have conflict of interest.

\section{Acknowledgements}

The authors wish to thank to all team from Recombinant Technology Laboratory/Bio-Manguinhos.

\section{Funding}

None.

\section{References}

1. World Health Organization. Pneumonia. Fact sheet $n^{\circ} 331$. Geneva, USA. 2016

2. Short K, Diavatopoulos D. Nasopharyngeal Colonization with Streptococcus pneumoniae, Streptococcus pneumoniae-molecular mechanism of host pathogen interactions, Academic Press, USA. 2015. p. 279-291.

3. Borrow R, Abad R, Trotter C, et al. Effectiveness of meningococcal serogroup C vaccine programmes. Vaccine. 2015;31(41):4477-4486.

4. World Health Organization. Pandemic \& Epidemic Diseases. Epidemic meningitis control -a three-pronged strategy to eliminate epidemic meningitis as a public health problem, Geneva, USA. 2015.
5. Chadwick S, Kriegel C, Amiji M. Nanotechnology solutions for mucosal immunization. Adv Drug Deliv Rev. 2010;62(4-5):394- 407.

6. Lycke N. Recent progress in mucosal vaccine development: potential and limitations. Nature. 2012;12:592-605.

7. Almada G, Haro C, Vintini E, et al. Safety of a nasal vaccine agaist Streptococcus pneumoniae using heat-killed Lactobacillus casei as adjuvant. Immunobiology. 2015;220(1):109-116.

8. Pereira IS. Intranasal formulation for conjugated meningococcal C vaccine, Master degree dissertation-Bio-Manguinhos, Oswaldo Cruz Foundation, Brazil. 2017.

9. Dutta PK, Dutta J, Tripathi VS. Chitin and chitosan: chemistry, properties and applications. Journal of Scientific \& Industrial Research. $2014 ; 63: 20-31$.

10. Kumar A, Vimal A, Kumar A. Why chitosan? From properties to perspective of mucosal drug delivery. Int $J$ Biol Macromol. 2016;91:615-622.

11. Casettari L, Vllasaliu D, Castagnino E, et al. PEGylated chitosan derivatives: synthesis, characterizations and pharmaceutical applications. Progress in Polymer Science . 2012;37(5):659-685.

12. Mouez MA, Zaki MN, Mansour Geneidi AS. Bioavailability enhancement of verapamil $\mathrm{HCl}$ via intranasal chitosan microspheres. Eur J Pharm Sci. 2014;51:59-66.

13. Cordeiro AS, Alonso MJ, Fuente M. Nanoengineering of vaccines using natural polysaccharides. Biotechnology Advances. 2015;33(6 Pt 3):1279-1293.

14. Xu JH, Dai WJ, Chen B Fan XY. Mucosal immunization with PsaA protein, using chitosan as a delivery system, increases protection against acute otitis nedia and invasive infection by Streptococcus pneumoniae. Scand J Immunol . 2015;81(3):177-185

15. Univ Leiden, Chiron SRL. Mucosal vaccine using chitosan adjuvant and meningococcal antigen. Giudice G Baudner Espacenet Patent. 2010. 